EPJ Web of Conferences 73, 02015 (2014)

DOI: $10.1051 /$ epjconf/20147302015

(C) Owned by the authors, published by EDP Sciences, 2014

\title{
Investigating the proton structure through polarization transfers in real Compton scattering processes at JLAB
}

\author{
C. Fanelli ${ }^{1,2, a}$, E. Cisbani ${ }^{3}$, D. Hamilton ${ }^{4}$, G. Salmè ${ }^{1,2}$ and B. Wojtsekhowski ${ }^{5}$ \\ ${ }^{1}$ Dipartimento di Fisica, Università La Sapienza, Rome, Italy \\ ${ }^{2}$ INFN, Sezione di Roma, 00185, Rome, Italy \\ ${ }^{3}$ INFN, Sezione di Roma, gruppo Sanità and Istituto Superiore di Sanità, 00161 Rome, Italy \\ ${ }^{4}$ Glasgow University, Glasgow, G12 8QQ, Scotland, UK \\ ${ }^{5}$ Thomas Jefferson National Accelerator Facility, Newport News, VA 23606, USA
}

\begin{abstract}
In the present work, preliminary results of the analysis of polarization transfers in real Compton scattering (RCS) performed in Hall-C at JLAB are presented, for data collected at large scattering angle $\left(\theta_{c m}=70^{\circ}\right)$ in the experiment E07-002. A previous experiment, E99-114, has already demonstrated a complete inconsistence of its results with a pQCD mechanism at JLAB energy regime. This kind of experiment, therefore, is of crucial importance for understanding the nature of the reaction mechanism for such a simple process, involving a real photon. Furthermore, RCS experiments allow the extraction of Compton form factors and Generalized Parton Distributions (GPDs), the latter connected to the total angular momentum of the nucleon.
\end{abstract}

\section{Physics motivation and preliminary results}

Real Compton scattering (RCS) in the hard scattering limit is a powerful probe of the structure of the nucleon. With a real photon at wide angle regime [1], RCS provides access to the high-t transverse structure of the hadron, whereas deep virtual Compton scattering (DVCS) provides access mostly to the high- $\mathrm{Q}^{2}$ low-t structure. For RCS on proton, the hard scale is reached when the Mandelstam variables $s,-t$, and $-u$ are larger than the proton mass. Under such conditions the transition amplitude is expected to factorize as $\mathrm{T}_{i f}(\mathrm{~s}, \mathrm{t})=\Psi_{f} \otimes \mathrm{K}(\mathrm{s}, \mathrm{t}) \otimes \Psi_{i}$, where $\mathrm{K}(\mathrm{s}, \mathrm{t})$ is the perturbative hard scattering amplitude, involving the coupling of the external photons to the active quarks, and the $\Psi$ 's are the soft initial and final wave functions, describing the coupling of the active quarks to the proton. Two different approaches are usually taken as a reference in the interpretation of the results: the "handbag" mechanism [2, 3], which involves only one active constituent (Fig. 1 (left)), with the soft physics for the coupling of this active quark to the proton described in the Generalized Parton Distributions (GPD's) [4, 5], and the "perturbative QCD” (pQCD) mechanism [6], in which all three valence quarks are active participants in the hard subprocess, the latter effected by the exchange of two hard gluons (Fig. 1 (right)), with the soft physics contained in the valence quark distribution. In principle, both mechanisms contribute to

\footnotetext{
ae-mail: cfanelli@iss.infn.it
}

This is an Open Access article distributed under the terms of the Creative Commons Attribution License 4.0, which permits unrestricted use, distribution, and reproduction in any medium, provided the original work is properly cited. 
Figure 1. Left: handbag diagram (crossed diagram must be considered); Right: $\mathrm{pQCD}$ diagram with two-gluon exchange $\mathrm{pQCD}$ diagram (plus 336 similar diagrams).
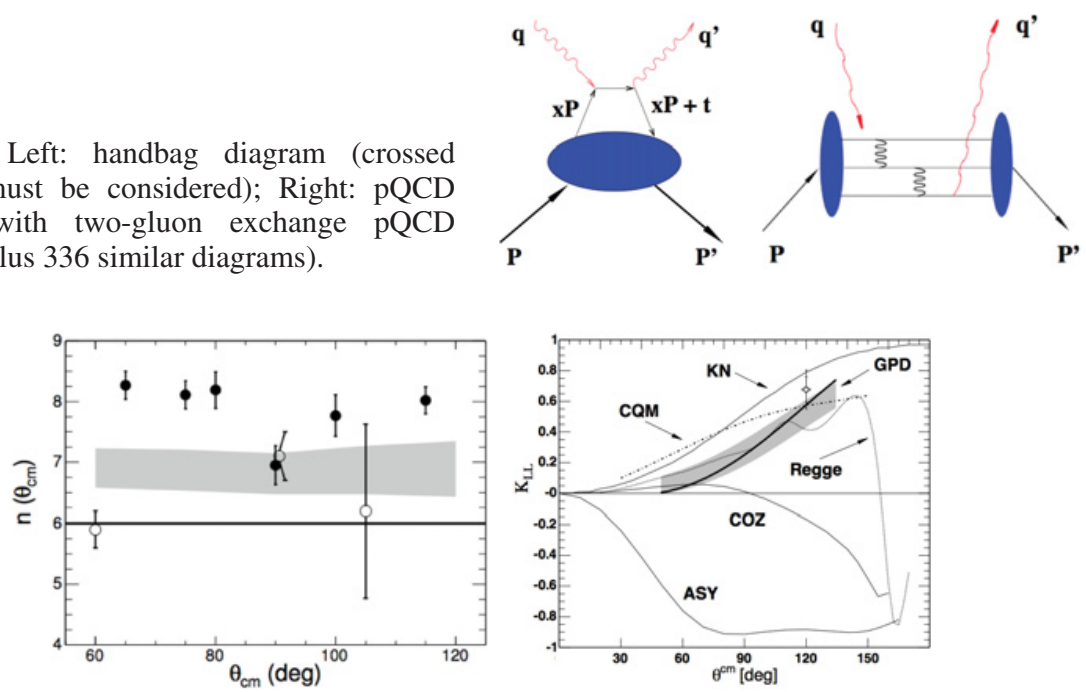

Figure 2. Left: scaling of the RCS cross section at fixed scattering angle $\theta_{c m}$. Full symbols denote the data from the E99-114 experiment [7]. The solid line corresponds to a constituent counting rule and scaling with fixed value $\mathrm{n}=6$. Open symbols denote the data from the Cornell experiment [10]. The gray band shows the range allowed by the GPD-based calculations according to [3]; Right: longitudinal polarization transfer in RCS vs $\theta_{c m}$. The point is the result of E99-114 at an incident photon energy of $3.2 \mathrm{GeV}$, at $\theta_{c m} \sim 120^{\circ}$ [9]. The curves represent theoretical prediction based on: KN (Klein-Nishina) for the asymmetry in the hard subprocess; GPD, shown as a gray band, for the handbag approach using GPD's [11]; CQM (constituent quark model) for the handbag approach [12]; Regge for a Regge exchange mechanism [13]; and COZ (Chernyak-Ogloblin-Zhitnitsky) and ASY (asymptotic) for pQCD calculations [14] using the ASY or COZ distribution amplitudes.

the scattering amplitude: at high energy, pQCD is expected to dominate, whereas at low energy, RCS is likely dominated by the non-perturbative mechanism. RCS experiment E99-114 [7] at JLAB has obtained two remarkable results, both showing strong inconsistence with the predictions of pQCD: (i) the scaling power for the s dependence of the RCS cross section at fixed center-of-mass angle $\left(\theta_{\mathrm{cm}}\right)$ was found to be $8.0 \pm 0.2$ [8] (Fig. 2 (left)), and (ii) the measurement (Fig. 2 (right)), at a single kinematical point [9], of the polarization transfer to the recoil proton by using longitudinally polarized incident photons, defined as:

$$
K_{L L}=\frac{1}{2}\left[\frac{\frac{d \sigma(\uparrow \uparrow)}{d t}-\frac{d \sigma(\uparrow \downarrow)}{d t}}{\frac{d \sigma(\uparrow \uparrow)}{d t}+\frac{d \sigma(\uparrow \downarrow)}{d t}}-\frac{\frac{d \sigma(\downarrow \uparrow)}{d t}-\frac{d \sigma(\downarrow \downarrow)}{d t}}{\frac{d \sigma(\downarrow \uparrow)}{d t}+\frac{d \sigma(\downarrow \downarrow)}{d t}}\right]
$$

where in the differential cross sections $\frac{d \sigma}{d t}$, the first arrow refers to the incident photon and the second to the recoil proton helicities. Several interesting issues demanded for further exploration of the RCS process: (i) does the RCS reaction proceed through the interaction of the photon with a single quark? (ii) what information can be obtained about the structure of the proton from new measurements of the RCS form factor $\mathrm{R}(\mathrm{t})$ ? (iii) at what kinematic scale does $\mathrm{pQCD}$ scaling begin to be predictive? Therefore, additional experimental investigations are needed, especially for the extraction of $\mathrm{K}_{L L}$, since E99-114 measurement was performed at a single kinematic point. To this purpose, in the JLAB Hall-C the RCS experiment E07-002 has been realized at a different scattering angle of 70 . The setup of E07-002, which ran in 2008 at Hall C, is described in Fig. 3.

The analysis can be divided into two parts: i) selection of RCS events and ii) extraction of polarization asymmetries (details can be found in [15]). The dipole magnet in front of the calorimeter deflects most of the electrons, therefore the main background sources are basically pion photoproduction, when the di-photon decay is misidentified as one photon, and Bethe-Heitler events, when a 
Figure 3. RCS experiment E07-002: a polarized photon beam, produced by bremsstrahlung of a polarized electron beam crossing a $6 \%$ copper radiator, is scattered from a liquid hydrogen target, transferring polarization to the recoiling protons. The protons are detected in a High-Momentum Spectrometer (HMS). A focal plane polarimeter (FPP), measures the polarization of the recoiling protons: two blocks of $\mathrm{CH}_{2}$ are used as analyzers, and tracking detectors are placed after each block to measure the azimuthal asymmetry in the angular distribution produced by spin-orbit coupling in the blocks (see also Fig. 4 (left)). The scattered photon is detected in a large acceptance electromagnetic calorimeter. Scattered electrons can also reach the calorimeter and constitute one of the main sources of background, but are largely identified by a dipole magnet in front of the calorimeter.
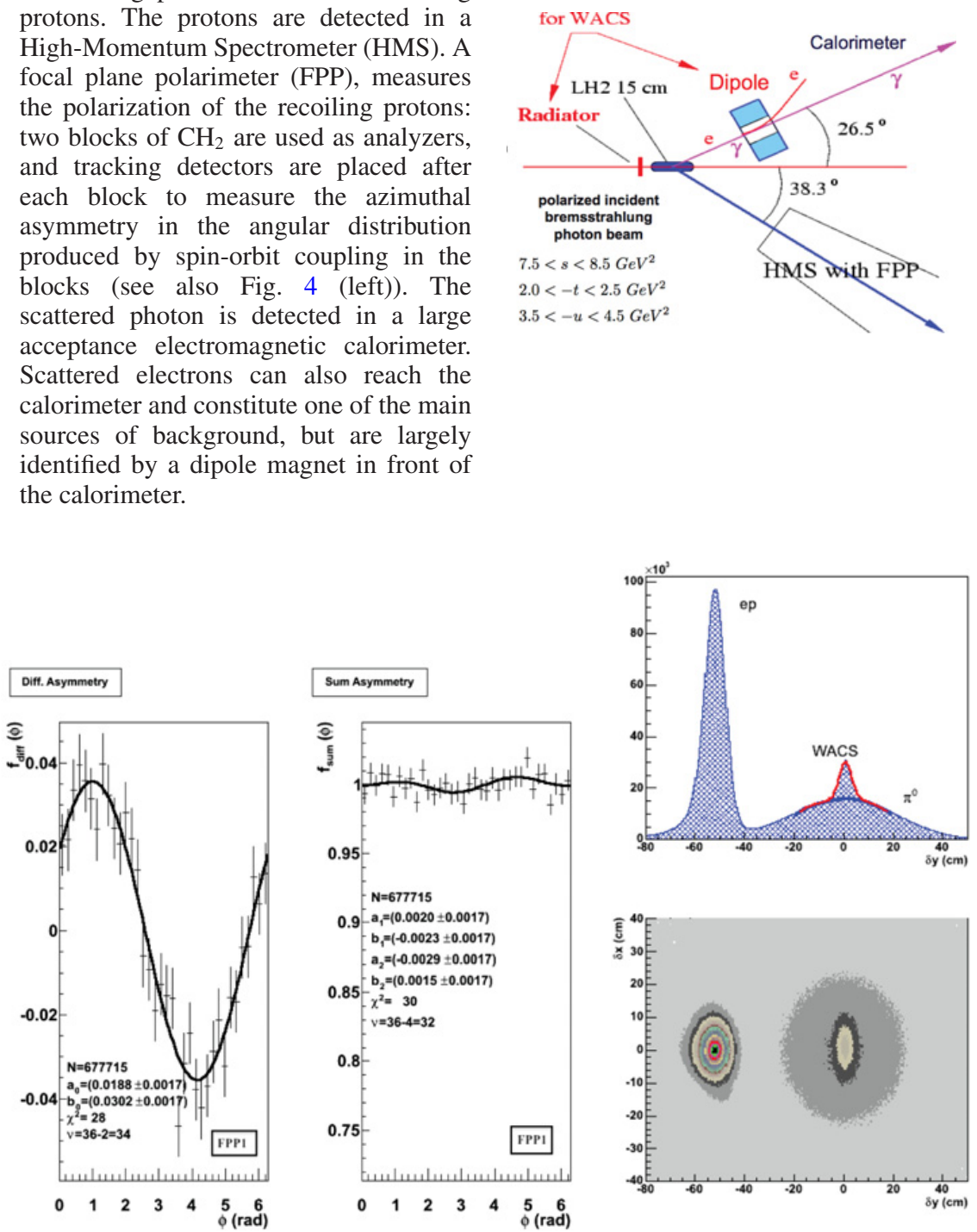

Figure 4. Left: difference and sum of helicity correlated distributions at $1^{\text {st }}$ focal plane of polarimeter (FPP1); Right: dilution factor $f$ estimated by the shape of the observable $\delta y$ defined in the text.

radiative photon in ep-scattering is emitted before the magnet. The RCS events of interest are located in a small elliptical region around $\delta \mathrm{x}=\delta \mathrm{y}=0$ in the calorimeter (difference between measured and predicted position of the photon in 2-body kinematics), contaminated mostly by pion events. A dilution factor can be estimated by the shape of the observable $\delta \mathrm{y}$, as $f \sim \mathrm{N}_{R C S} / \mathrm{N}_{t o t}$, cf. Fig. 4 (right). The final longitudinal polarization transfer, can be expressed as: $K_{L L}^{R C S}=\frac{1}{f}\left[K_{L L}^{t o t}-(1-f) K_{L L}^{\pi}\right]$. 
Figure 5. Preliminary result for $\mathrm{K}_{L L}$ at $\theta_{c m}=$ $70^{\circ}, Q^{2}=2.1 \mathrm{GeV}^{2}$, and incident mean energy of $3.8 \mathrm{GeV}$. Refer to Fig. 2 for the theoretical curves CQM, GPD, COZ, Regge and ASY.

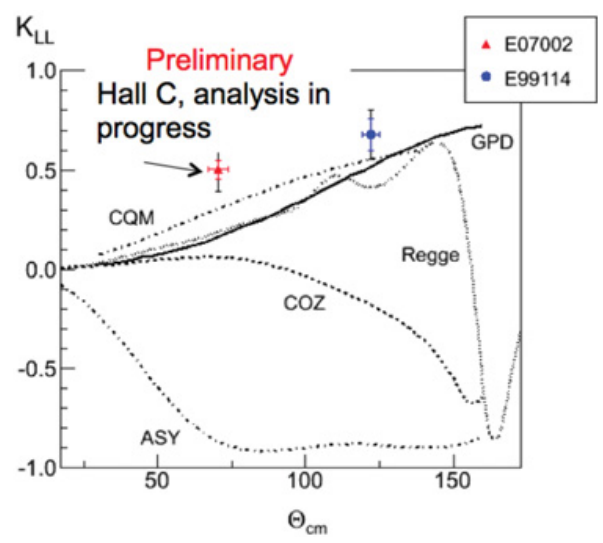

\section{Conclusions}

Recent advancements in the analysis of the polarization transferred to the recoil proton in RCS have been reported in this work, at $\theta_{c m}=70^{\circ}$. At these kinematics the preliminary results reported in Fig. 5 seems to exclude pQCD based predictions, in agreement with the previous published result of experiment E99-114 [9] at higher angle. The optimization of the selection criteria, the estimation of the BetheHeitler contamination, and finally a full study of systematics are still underway. The results of this analysis and future experiments at JLAB, at larger beam energies available and over a broader kinematic range, will shed light on the structure of the nucleon, allowing to individuate the dominant mechanism among non-perturbative and perturbative approaches. These RCS data will allow to understand how the factorization is realized, and will be a potential source of information for the understanding of the two-photon exchange effects in elastic electron-nucleon scattering.

We thank W. Luo for his precious and continuous support of the data analysis and E07-002 collaborators.

\section{References}

[1] N. Kivel and M. Vanderhaeghen, JHEP 1304, 029 (2013); [arXiv: 1212.0683]

[2] M. Diehl and T. Feldmann and R. Jakob and P. Kroll, Eur. Phys. J. C 8, 409-434 (1999)

[3] H.W. Huang and P. Kroll and T. Morii, Eur. Phys. J. C 23, 301-310 (2002)

[4] X. Ji, Phys. Rev. D 55, 7114 (1997), Phys. Rev. Lett. 78, 610 (1997)

[5] A.V. Radyushkin, Phys. Lett. B 380, 417 (1996), Phys. Rev. D 56, 5524 (1997)

[6] R. Thomson, A. Pang and C.R. Ji, Phys. Rev. D 73, 054023 (2006)

[7] C. Hyde-Wright, A. Nathan and B. Wojtsekhowski (spokespersons), JLab experiment E99-114

[8] A. Danagoulian et al. (Jefferson Lab Hall A Collaboration), Phys. Rev. Lett. 98, 152001 (2007)

[9] D.J. Hamilton, V.H. Mamyan et al., Phys. Rev. Lett. 94, 242001 (2005)

[10] M.A. Shupe et al., Phys. Rev. D 19, 1921 (1979)

[11] M. Diehl et al., Phys. Rev. D 67, 037502 (2003)

[12] G.A. Miller, Phys. Rev. C 69, 052201(R) (2004)

[13] F. Cano and J.M. Laget, Phys. Rev. D 65, 074022 (2002)

[14] T. Brooks and L. Dixon, Phys. Rev. D 62, 114021 (2000)

[15] A.J.R. Puckett, Ph.D. thesis (2010) 